\title{
РАЗРАБОТКА СИСТЕМЫ ИНДИКАТОРОВ ОЦЕНКИ ОТРАСЛЕВЫХ РИСКОВ В ПРОЦЕССЕ РАСХОДОВАНИЯ СРЕДСТВ НА ФЕДЕРАЛЬНЫЕ ПРОЕКТЫ
}

\author{
(C) 2021 Петров Александр Михайлович \\ доктор экономических наук, профессор Департамента бизнес-аналитики \\ Финансовый университет при Правительстве Российской Федерации, Россия, Москва \\ профессор кафедры бухгалтерского учета и налогообложения \\ Российский экономический университет им. Г. В.Плеханова, Россия, Москва \\ E-mail: palmi@inbox.ru
}

Информация, используемая экономическим субъектом, должна быть полезна для снижения последствий будущих угрожающих событий. Угрожающие события могут возникнуть по причине внешних источников, к примеру, резкое изменение рыночной ситуации или новые требования регулирующих органов или законодательства. Таким образом, многие экономические субъекты определяют, что причинами возникновения нежелательных событий в большей степени являются внешние факторы. Поэтому необходима разработка системы индикаторов оценки отраслевых рисков, чему и посвящена статья.

Ключевые слова: бюджетные нарушения, федеральный проект, отраслевой риск, ключевые индикаторы риска.

Внешние источники информации (публикации СМИ или рейтинговых агентств) могут быть полезны при формировании системы индикаторов. Опыт взаимодействия, мониторинг действий конкурентов или интервьюирование клиентов, заказчиков, подрядчиков, сотрудников, поставщиков может дать ключевое понимание рисковой области, с которой столкнулся субъект хозяйствования. Понимание нормативных и законодательных требований и особенностей отрасли позволит субъекту хозяйствования оперативно и заблаговременно реагировать на потенциальные угрозы. Внутренние источники информации, наоборот, в некоторых случаях бывают субъективными, но только они одни могут быть доступны в текущий период времени для прогнозирования и предотвращения воздействия возможных нежелательных событий.

Низкая вероятность того, что один ключевой индикатор риска может отражать все стороны угрожающего события. Поэтому, необходимо отслеживать комплекс ключевых индикаторов деятельности, которые могут подавать сигналы о возникновении риска. При анализе такого комплекса необходимо учитывать степень влияния каждого индикатора деятельности в структуре риска и разделять эти индикаторы по весовым шкалам в зависимости от степени влияния. Необходимо агрегировать весь комплекс информации, самым лучшим способом обеспечивающей предварительную диагностику и предотвращение угроз. Регулярные анализ, осмысление и опыт позволяют сделать наиболее значимые выводы и повысить эффективность ключевых индикаторов риска. Для формирования оптимального набора индикаторов риска необходимо проводить их регулярную актуализацию и развитие, а также определить принципы эффективного формирования ключевых индикаторов риска. На рисунке 1 приведены основные принципы эффективного формирования ключевых индикаторов риска.

На основе описанной выше модели оценки отраслевых рисков и изученных принципов и подходов к эффективному формированию ключевых индикаторов риска авторами разработана модель ключевых индикаторов отраслевых рисков, исходя из реалий текущих угроз функционирования субъектов хозяйствования.

Исходя из допущения того, что основной стратегической целью деятельности субъекта хозяйствования является получение прибыли, авторами принимаются описанные выше идеи о том, что хозяйствующие субъекты осуществляют свою деятельность для увеличения доходности в будущем, в соответствии с чем, можно выделить основные задачи субъекта хозяйствования на сегодняшний день: стабильное обеспечение потребителей продукцией, выпускаемой хозяйствующим субъектом, в соответствии с 


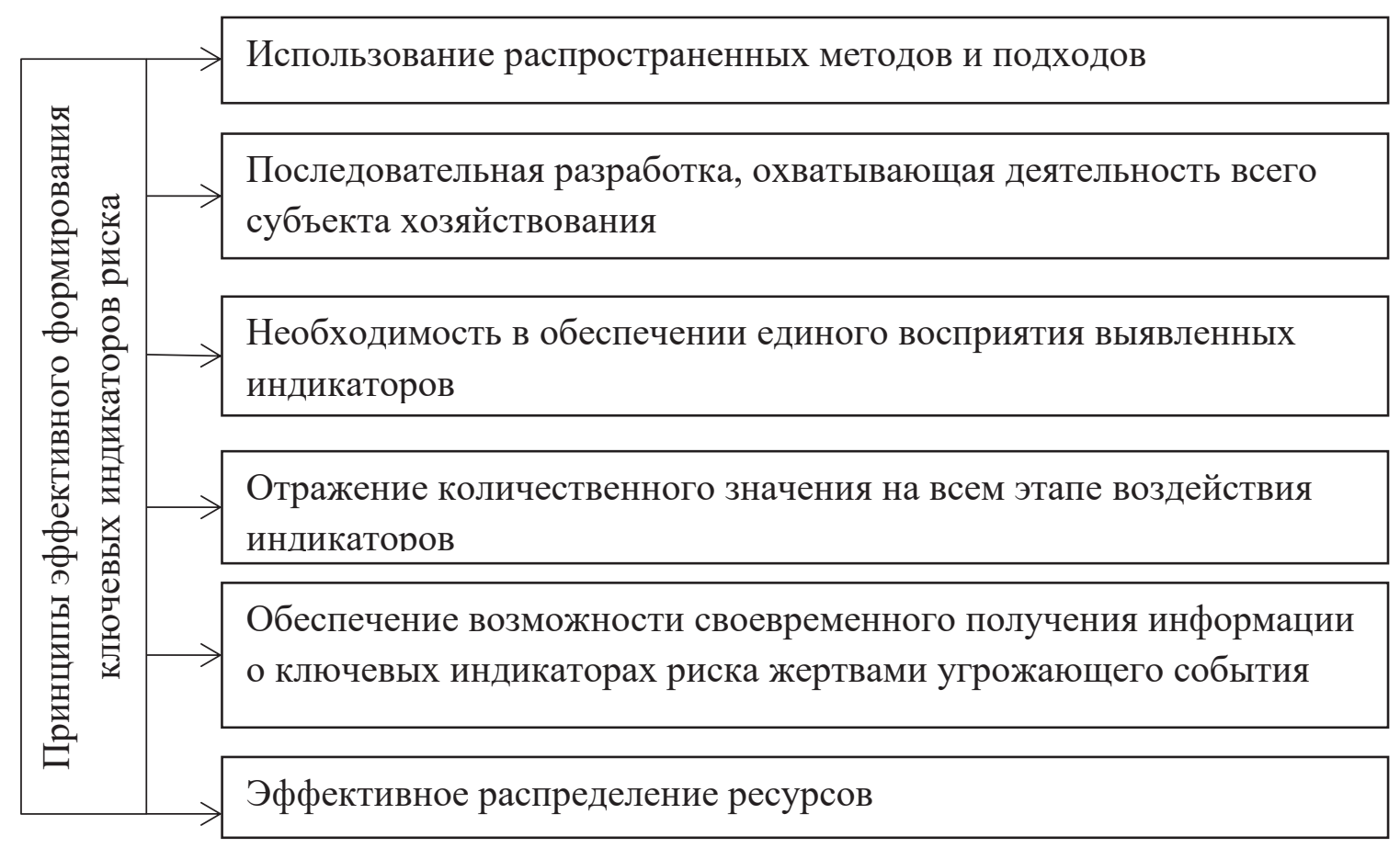

Рисунок 1. Принципы эффективного формирования ключевых индикаторов риска

заключенными контрактами и рыночным спросом; недопущение сбоев в производственном процессе; обеспечение персонала заработной платой и привлекательными условиями труда, а также возможностью профессионального роста; охрана экологии окружающей среды.

Однако для российских экономических субъектов в 2020 году возникают значительные внешние и внутренние риски, которые ставят под угрозу выполнение вышеописанных стратегических задач деятельности и функционирования экономического субъекта в целом.

Главной угрозой для отраслей производства в 2020 году становится пандемия, в результате которой были остановлены или переведены на дистанционную работу большинство бизнессубъектов. Несмотря на меры поддержки бизнеса, которые активно принимаются региональными и федеральными властями, значительно страдают единицы крупного бизнеса в России, а также большой урон нанесен малому и среднему бизнесу.

К отраслям, которые наиболее пострадали от кризиса, вызванного пандемией 2020 года, относится туристическая отрасль, которая фактически на сегодняшний день не функционирует. Правительство РФ предпринимает активные шаги по развитию внутреннего туризма, кото- рый может стать драйвером роста туристической отрасли. Кроме этого, значительные угрозы для дальнейшего функционирования остаются у сектора фитнеса и индустрии красоты и здоровья, так как на длительный период были закрыты фитнес-центры, бассейны, спортивные клубы. Рост заболеваемости населения страны может повлечь за собой очередное закрытие фитнес-центров, бассейнов, спортивных клубов. Сильно страдает ресторанный бизнес и структуры, связанные с организацией массовых мероприятий. Также упадок ждет потребительскую отрасль (кроме продуктовой и аптечной сети) в связи с тем, что люди меньше ходят в магазин и в большие торгово-развлекательные центры за покупками в целом из-за падения доходов населения. Кроме этого, в отношении Российской Федерации действуют антироссийские санкции, введенные США и рядом Европейских стран. В режиме действия таких мер инвестиционная привлекательность российского бизнеса снижается и с каждым годом инвестиционный поток только уменьшается.

По итогам 2019 года существенно сократился глобальный рост мировой экономики в целом. Замедление экономики является следствием кризиса потребления.

Еще одним риском для отраслей российской 
экономики является усугубление торговых конфликтов из-за трудностей, возникающих между США и Китаем. Данный конфликт приводит к торговому дисбалансу и волатильности цен на сырьевых рынках. Этот фактор оказывает значительное влияние на внутреннюю экономику России, в связи с ее зависимостью от цен на энергоносители. Таким образом, на примере схемы выявления ключевых индикаторов риска в исследовании построена система индикаторов оценки отраслевых рисков, иллюстрирующая актуальную на сегодняшний день ситуацию в российской экономике.

«Индикатор риска 1» представляет собой снижение прибыли от продаж продукции экономических субъектов при остающемся высоком уровне рыночного спроса. Прибыль от продаж является одним из важнейших показателей, которые характеризуют эффективность деятельности экономического субъекта. В общем виде этот показатель вычисляется как разница между денежными средствами, полученными от продаж, и затратами предприятия. На этот показатель оказывают влияние факторы: изменение ценовой политики, снижение или увеличение объема выручки, увеличение или уменьшение затрат, повышение или уменьшение себестоимости производимой продукции.

В связи с тем, что показатель прибыли субъекта хозяйствования, является основным показателем его финансовой рентабельности, уменьшение прибыли от продаж может свидетельствовать о финансовых трудностях субъекта хозяйствования и низкой эффективности хозяйственной деятельности (рисунок 2).

Еще одним индикатором отраслевого риска будет являться снижение показателя чистой прибыли при сокращении производств. С учетом возникших в 2020 году экономических трудностей для субъектов хозяйствования, в особенности малого и среднего бизнеса, большинство отраслей потеряет значительную долю чистой прибыли в сравнении с показателями 2019 года. При оставшемся рыночном спросе на уровне прошлого года, снижение показателя чистой прибыли будет означать только сокращение производств вследствие двух основных факторов: отсутствие финансирования или сокращения производства.

Основную долю материальных затрат пред-

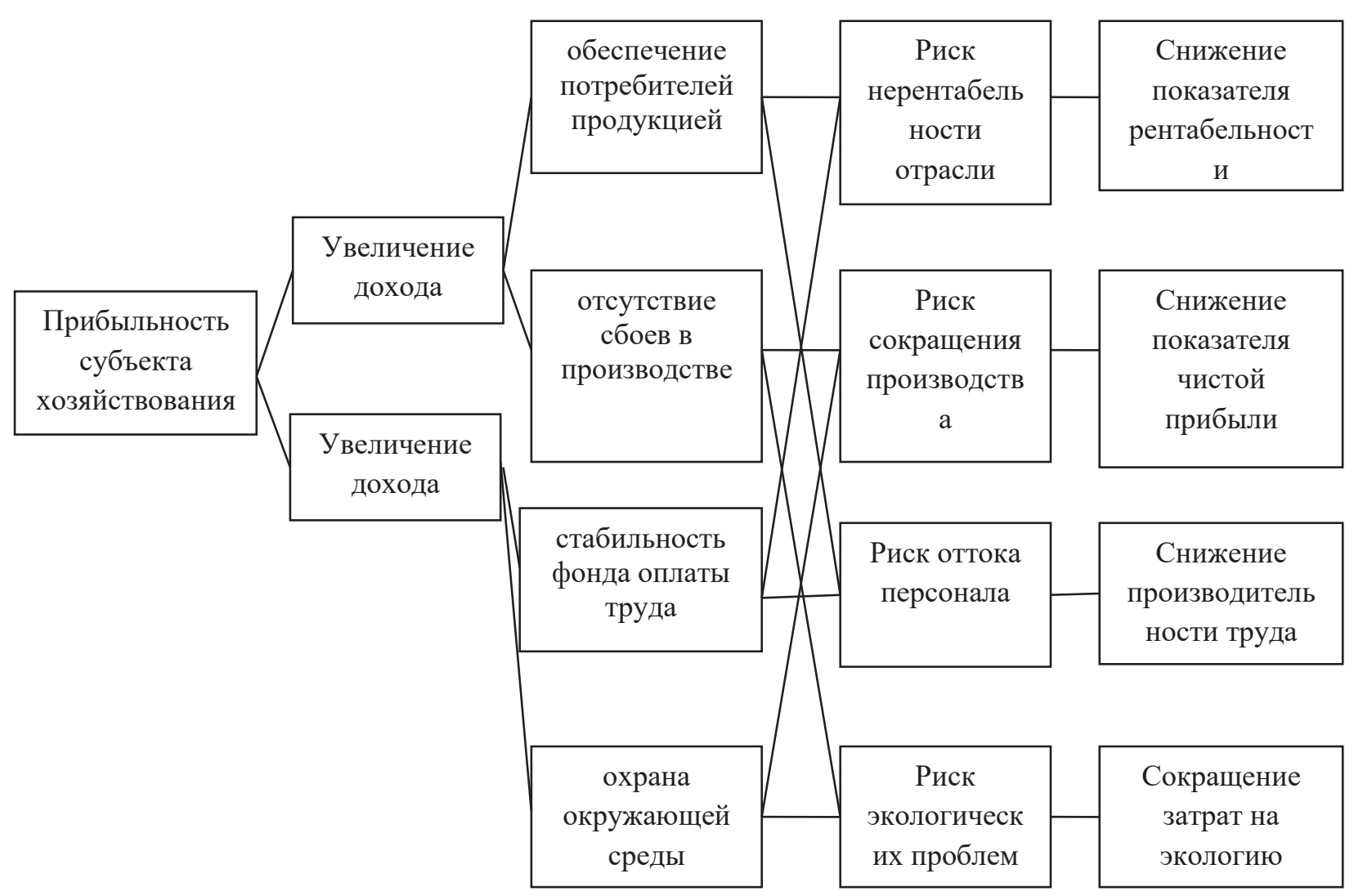

Рисунок 2. Схема ключевых индикаторов отраслевого риска субъекта хозяйствования 
приятий любых отраслей занимает показатель расходов на оплату труда. Вследствие возникающих угроз и развития нежелательных ситуаций, показатель расходов на оплату труда будет сокращаться, что приведет к оттоку персонала. Для предприятий большинства российских отраслей отток персонала будет означать, опять же, сокращение производства, что, в конечном счете, приведет к риску нерентабельности отрасли и ее исчезновению.

Приоритизация индикаторов риска может помочь пользователям информации уделять внимание наиболее значимым индикаторам для субъекта хозяйствования.

Определение приоритетов можно автоматизировать через использование предельных уровней, но в этом случае требуется оперативное решение ответственного лица за оценку риска. Экономическим субъектам также следует рассмотреть вопрос о согласовании некоторых общих критериев для определения степени приоритетности индикаторов риска, учитывая при этом, что уровень приоритетности может быть различным в соответствии уровню, на котором индикаторы рассматриваются в рамках субъекта хозяйствования. Например, в крупных экономических субъектах уровень значимости может быть выше на общем уровне, в связи с этим, на разных уровнях управления, возможно, потребуется согласовать различные критерии для определения приоритетности тех или иных индикаторов и связанных с ними рисков.

Для созданной модели ключевых индикаторов отраслевых рисков, индикаторами «красной зоны» значений индикаторов будут такие индикаторы, как «снижение показателя рентабельности» и «снижение показателя чистой прибыли». Если значения этих индикаторов слишком низкие, риск-менеджеру необходимо будет сделать вывод о подверженности субъекта хозяйствования значительному риску. В таком случае со стороны руководства необходимо срочное принятие решений по устранению нежелательных событий.

Кроме этого, может быть еще также «желтая зона» и «зеленая зона» индикаторов. Значение индикатора «желтой зоны» предполагает отнесение субъекта хозяйствования к среднему уровню риска, а значит, субъекту хозяйствования необходимо определить величину угрозы опасного события данного индикатора и необходимость принятия мер. Значение индикатора «зеленой зоны» означает нахождение индикатора в пределах нормы, что означает отсутствие приверженности субъекта хозяйствования данному риску. К индикатору «зеленой зоны» можно отнести индикатор «сокращение затрат на экологию».

Исследование подходов к оценке отраслевых рисков, а также разработка системы индикаторов оценки отраслевых рисков будет способствовать разработке эффективного инструментария в части контроля использования бюджетных средств, выделенных на федеральные проекты.

Выводы.

В научно-исследовательской работе проведено ранжирование возможных нарушений при расходовании средств на федеральные проекты, исследованы подходы к оценке отраслевых рисков и разработана система индикаторов оценки отраслевых рисков в процессе расходования средств. Обусловлена необходимость применения различных методов оценки риска в практике деятельности субъектов хозяйствования и дана характеристика их возможного применения. В исследовании дана собственная точка зрения на методы управления рисками, формирование системы индикаторов и процесс отбора ключевых индикаторов риска. Выявлены принципы эффективного формирования ключевых индикаторов риска.

\section{Библиографический список}

1. Федеральный закон от 26.07.2006 N 135-Ф3 (ред. от 24.04.2020) «О защите конкуренции» // СПС КонсультантПлюс.

2. Райзберг, Б. А. Современный экономический словарь / Б. А. Райзберг, Л.Ш. Лозовский, Е. Б. Стародубцева. 6-е изд., перераб. и доп. - Москва: ИНФРА-М, 2017. - 512 с.

3. Петрова О.А. Проблемы развития рынка факторинговых услуг / - Экономические науки, № 9 (202), 2021. С. 232-234

4. Chernysheva N.A., Perskaya V.V., PetrovA.M., Bakulina A.A. Green energy for belt and road initiative: economic aspects today and in the future / International Journal of Energy Economics and Policy. 2019. T. 9. № 5. C. 178-185. 
5. Kevorkova Z.A., Petrov A. M., Savina N. V. TOWARDS LIABILITIES OF CORPORATE SYSTEMS / International Journal of Civil Engineering and Technology. 2019. T. 10. № 2. C. 1582-1593.

6. Petrov A.M., Nikiforova E.V., Kiseleva N.P., Grishkina S. N., Lihtarova O.V. CREATION OF THE REPORTING ON SUSTAINABLE DEVELOPMENT OF COMPANIES BASED ON SOCIOECONOMIC MEASUREMENT STATISTICS / International Journal of Recent Technology and Engineering. 2019. T. 8. № 2. C. 4005-4012.

7. Sotnikova L.V., Polenova S.N., Mislavskaya N.A., PetrovA.M., BasovaM.M. SUSTAINABle DeVELOPMENT, MACRO AND MICRO LEVEL: RUSSIAN AND FOREIGN MODEL / International Journal of Recent Technology and Engineering. 2019. T. 8. № 2. C. 4524-4532.

8. Kosolapova M.V., Muravitskaya N.K., Tolmachev M. N., Melnikova L.A., PetrovA.M. TECHNOLOGY FOR SOLVING THE PROBLEMS RELATED TO THE IMPLEMENTATION OF THE CONCEPT OF PRESERVING CAPITAL IN ACCOUNTING AND STATISTICS / International Journal of Recent Technology and Engineering. 2019. T. 8. № 3. C. 789-792.

9. Kosolapova M.V., PetrovA.M., YshanovI.G., Muravitskaya N.K., NurmuhamedovaH.S. THE ECONOMIC SIGNIFICANCE OF STATISTICAL RESEARCH ACTIVITIES OF REPRESENTATIVE OFFICES OF COMPANIES ABROAD / International Journal of Innovative Technology and Exploring Engineering. 2019. T. 8. № 10. C. 27132722.

10. Petrov A.M., Yurasova I. O., Putihin Y.E., Poluleh M. V., Erohina V.N. ACCOUNTANT MODELING TECHNOLOGY AND STATISTICS IN THE CONTEXT OF THE NEW EDUCATIONAL CONCEPT / International Journal of Innovative Technology and Exploring Engineering. 2019. T. 8. № 12. C. 3214-3217.

11. Petrov A.M., Kiseleva N.P., Kevorkova Z.A., Melnikova L.A., YshanovI.G. PRESENT DEVELOPMENT PRACTICES FOR TAX, FINANCIAL AND STATISTICAL REPORTING IN THE RUSSIAN FEDERATION / International Journal of Innovative Technology and Exploring Engineering. 2019. T. 8. № 12. C. 3538-3542.

12. Karpova T.P., Petrov A.M., Antonova O.V. DIRECTIONS OF ACCOUNTING DEVELOPMENT IN THE CONDITIONS OF DIGITALIZATION / Journal of Advanced Research in Dynamical and Control Systems. 2018. T. 10. № 7 Special Issue. C. $117-125$

13. Lymar M.P., Kevorkova Z.A., Petrov A. M. THE CONVERGENCE OF NATIONAL AND INTERNATIONAL ACCOUNTING STANDARDS: CHINESE EXPERIENCE / International Journal of Civil Engineering and Technology. 2018. T. 9. № 13. C. 82-94.

14. Бабаев Ю.А., Друцкая М.В., Кеворкова Ж. А., Листопад Е. Е., Петров А. М. БУХГАЛТЕРСКИЙ УЧЕТ, АНАЛИЗ И АУДИТ ВНЕШНЕЭКОНОМИЧЕСКОЙ ДЕЯТЕЛЬНОСТИ / учебник для студентов обучающихся по специальности 080109 «Бухгалтерский учет, анализ и аудит» / под редакцией Ю.А. Бабаева. Москва, 2010.

15. Петров А.М., Полоус Е. А. ПОВЫШЕНИЕ ТРАНСПАРЕНТНОСТИ ПОКАЗАТЕЛЯ ДЕБИТОРСКОЙ ЗАДОЛЖЕННОСТИ В ОТЧЕТНОСТИ / Международный бухгалтерский учет. 2011. № 6 (156). С. 2-12.

16. Карпова Т.П., Петров А. М., Горбаткова Г.А., Самарина Л. Б., Дашкина Г.Г., Сидорова М. И., Сабанин Р. Л., Ситникова В. А., ЛИстопад Е. Е. БУХГАЛТЕРСКИЙ УЧЕТ В СФЕРЕ УСЛУГ / Учебник для студентов высшего профессионального образования, обучающихся по специальности 080109 «Бухгалтерский учет, анализ и аудит» / Под редакцией М.А. Вахрушиной; Москва, 2011. Сер. Читай

17. Петров А.М., МельнИковаЛ.А. ФОРМИРОВАНИЕ ОТЧЕТНОСТИ В СООТВЕТСТВИИ С ТРЕБОВАНИЯМИ МСФО КАК ОБЪЕКТИВНАЯ НЕОБХОДИМОСТЬ НА СОВРЕМЕННОМ ЭТАПЕ РАЗВИТИЯ ЭКОНОМИКИ РФ / Проблемы современной экономики. 2017. № 2 (62). С. 105-107.

18. ТЕОРИЯ БУХГАЛТЕРСКОГО УЧЕТА / учебник / Ю.А.Бабаев, А. М.Петров; под ред. Ю.А.Бабаева. Москва, Проспект, 2011. (Изд. 5-е, перераб. и доп.) 